\title{
Does the User's Evaluation of a Socially Assistive Robot Change Based on Presence and Companionship Type?
}

\author{
Sebastian Schneider \\ Applied Informatics \\ CITEC, Bielefeld University \\ 33615 Bielefeld \\ Bielefeld, Germany \\ sebschne@techfak.uni-bielefeld.de
}

\author{
Franz Kummert \\ Applied Informatics \\ CITEC, Bielefeld University \\ 33615 Bielefeld \\ Bielefeld, Germany \\ franz@techfak.uni-bielefeld.de
}

\begin{abstract}
A lack of motivation is the most common obstacle for physical activity. Socially Assistive Robots (SAR) can be used to motivate people to workout regularly. However, the embodiment and companionship type can influence user's engagement. We investigated the effects of embodiment (co-present vs. remote-located) and companionship (instructor vs. companion) in a video Human-Robot Interaction (vHRI) study $(\mathrm{n}=90)$.
\end{abstract}

\section{Keywords}

Human-Robot Interaction; Embodiment; Social Assistance

\section{INTRODUCTION}

Regular physical activity is important for preventing obesity and other associated diseases. A lack of motivation is the most common obstacle for physical activity. Recently, there is a trend for using active video games to promote rehabilitation and physical activity [3]. These approaches seem to be a suitable addition to standard methods of rehabilitation. They provide accompanying virtual agents or mirror the actions of the user [2]. The shift of focus from workout to play in games is believed to increase people's and specially children's exercising motivation because they "capitalize on children's natural interest in computerized video interaction" [3]. However, the long-term effects of exergaming with entertainment systems is contented [3]. In our line of research, we question whether video games and virtual agents are sufficient to increase long-term motivation to exercise or whether embodiment is needed to increase the motivation. In contrast to agents in exergames, socially assistive robot (SAR) [6] are embodied in the real world and this embodiment has proven to enhance the user's engagement [4].

However, the choice of embodiment and presence is still an important aspect to consider and dependent on the robot's task. If the robot has to actually fetch and carry objects

Permission to make digital or hard copies of part or all of this work for personal or classroom use is granted without fee provided that copies are not made or distributed for profit or commercial advantage and that copies bear this notice and the full citation on the first page. Copyrights for third-party components of this work must be honored. For all other uses, contact the owner/author(s).

HRI '17, March 6-9, 2017, Vienna, Austria.

(C) 2017 Copyright held by the owner/author(s).

ACM ISBN 978-1-4503-4336-7/17/03.

DOI: http://dx.doi.org/10.1145/3029798.3038418 in the environment it is clear that a physical embodiment is required, but in the context of social assistance it is not evident whether a co-located robot has any advantage compared to a remote-located or virtual robot [4]. The primary research hypothesis of our current work is that exergames are missing a social component (i.e. embodiment) which is necessary to motivate people to workout. While it is challenging to build robots that can exercise co-actively with the user we are investigating how the robot's style of companionship influences the user's perception of the robot. Therefore, we question whether it is sufficient for a SAR to only instruct the users during a physical activity or whether they have to exercise co-actively. The results will lead to different requirements for engineers developing new robot platforms. Do future social robots need to exercise co-actively with their interaction partner or not? To investigate these questions, we implemented a study comparing the different effects between presence and companionship type.

\section{STUDY DESIGN}

To answer the mentioned questions we conducted a video Human-Robot Interaction (vHRI) study. We gathered the people's evaluation of different embodiment and companionship types of a robot in the context of a socially assistive workout scenario. We evaluated four different conditions: a remote-located instructor robot, a remote-located co-actively exercising companion robot, a co-located instructor robot and a co-located co-actively exercising companion robot. For each condition we recorded a video of 2 minutes length showing a robot interacting with a human during a sport exercise (see Fig. 1) and asked the participants to view the video and rate their perception of the robot. In the video the robot first introduces three exercises (e.g. jumping jacks, squats, side lunges) and then instructs the user to do the exercises. For all videos the robot behavior was exactly the same. We only manipulated the presence of the robot (displayed on a 42 inch screen vs. on located on a table) and whether the robot is only instructing or co-actively exercising with the user. We hypothesize that

H1: the co-located robot condition will be evaluated higher on all scales compared to the virtual conditions,

H2: the co-located robot companion will be evaluated higher on the likeability and animacy scales compared to the co-located instructor condition.

We acquired the participants through online social media and on our campus. The participants were randomly 


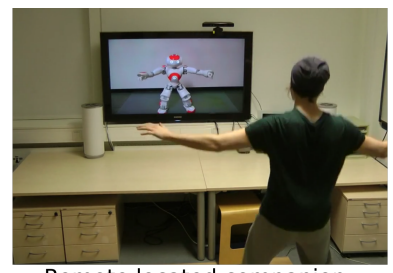

Remote located companion

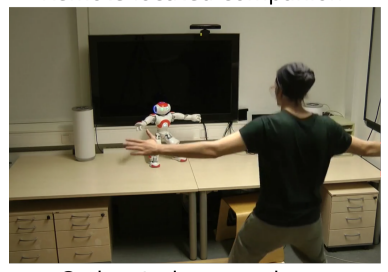

Co-located companion

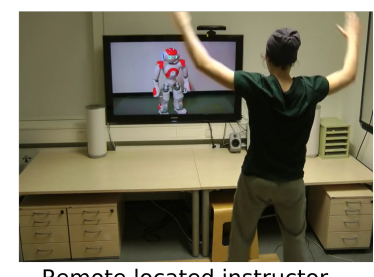

Remote located instructor

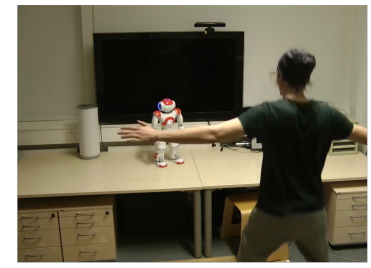

Co-located instructor

Figure 1: Stimulus material for the different conditions.

assigned to one of the four conditions. In total we had 90 participants with mean age $M=27.28$ and $S D=9.8$ (male: 38 , female 52). However, we needed to exclude 6 participants that did not finish the survey.

\subsection{Measures}

First, we assessed the user's exercising behavior and experience with technology (e.g computers, robots) on a 5point Likert-scale. Second, we measured the perception of the robot using the Godspeed questionnaire on a 5-point differential scale [1]. Third, we asked the participants to rate whether they wish to have an assistive coaching system. At last, we asked the participant to rate the role they would would ascribe to the robot using multiple choice answers (e.g. machine, toy, useless technology, partner, coach, teacher).

\section{RESULTS}

We conducted an analysis of variance between the groups for prior knowledge $(\mathrm{F}(1,84)=0.119$, n.s. $)$, exercising per week $(\mathrm{F}(1,84)=1.08$, n.s. $)$, wish for future assistance of a system $(\mathrm{F}(1,84)=0.681$,n.s. $)$ and gender $\mathrm{F}(1,84)=2.545$, n.s. $)$. We found a difference for the factor age $(\mathrm{F}(1,84)=4.243$, $p<.05)$. However, a pairwise comparisons using t-tests did not reveal any significant difference between the conditions (all $p$-values >.1). Hence, our randomization was successful.

We conducted a multivariate analysis of variance to find differences in the perception of the robot based on the Godspeed questionnaire. The internal consistency of the item sets is as follows: Intelligence: $\alpha=.81$, Anthropomorphism: $\alpha=.76$, Animacy: $\alpha=.86$, Likeability: $\alpha=.9$, Anxiety: $\alpha=.65$. Using a Pillai's trace, there was no significant effect found on all scales of the Godspeed Questionnaire between the four conditions $(\mathrm{V}=0.13, \mathrm{~F}(3,240)=0.75, \mathrm{p}=0.72)$.

Furthermore, we analyzed the difference regarding the perceived social role of the system using a $X^{2}$ analysis which also revealed no significant differences between the conditions.

\section{DISCUSSION}

Our primary objective of this research was to investigate whether the people's perception of different embodiment and social roles of a robot changes. To investigate these question

we conducted a vHRI study. The results of our work do not support our hypotheses. Hence, we want to propose explanations why we could not find evidence for our hypotheses and discuss some confounding factors. We suppose that the effect of the embodiment and role of the robot is hindered by several factors: a) The length of the video was too short (i.e. 2 minutes); b) The size of the robot is subjectively bigger in the remote-located conditions due to the size of the display than in the companion condition. This could influence the user's perception of the robot since taller persons have been found to be perceived more positively [5]; c) The appearance of both robots is too similar and both are likely to be perceived equally realistic. At last d) We can not control whether the people were watching the videos attentively.

\section{CONCLUSION}

Our study shows that VHRI experiments might not be suitable to investigate embodiment effects. Participants in the remote located conditions are exposed to a video of a simulation of a robot. Thus, the participant's perception of the robot's embodiment might be ambiguous and thus result in no differences. We suppose that the same argument applies for the missing difference regarding the companionship type of the robot. The participants are not interacting with the system and therefore they do not perceive any difference regarding the companionship type of the robot. Hence, our future work includes to investigate our proposed research questions in real HRI studies.

\section{Acknowledgments}

This research/work was supported by the Cluster of Excellence Cognitive Interaction Technology 'CITEC' (EXC 277) at Bielefeld University, which is funded by the German Research Foundation (DFG).

\section{REFERENCES}

[1] C. Bartneck, D. Kulić, E. Croft, and S. Zoghbi. Measurement instruments for the anthropomorphism, animacy, likeability, perceived intelligence, and perceived safety of robots. International journal of social robotics, 1(1):71-81, 2009.

[2] D. L. Feltz, S. T. Forlenza, B. Winn, and N. L. Kerr. Cyber buddy is better than no buddy: A test of the köhler motivation effect in exergames. GAMES FOR HEALTH: Research, Development, and Clinical Applications, 3(2):98-105, 2014.

[3] Z. Gao, S. Chen, D. Pasco, and Z. Pope. A meta-analysis of active video games on health outcomes among children and adolescents. obesity reviews, 16(9):783-794, 2015.

[4] J. Li. The benefit of being physically present: a survey of experimental works comparing copresent robots, telepresent robots and virtual agents. International Journal of Human-Computer Studies, 77:23-37, 2015.

[5] G. Stulp, A. P. Buunk, S. Verhulst, and T. V. Pollet. High and mighty: Height increases authority in professional refereeing. Evolutionary Psychology, 10(3):147470491201000314, 2012.

[6] A. Tapus, M. J. Mataric, and B. Scasselati. Socially assistive robotics [grand challenges of robotics]. Robotics E Automation Magazine, IEEE, 14(1):35-42, 2007. 